\title{
Interpreting Multiple Regression Results: $\beta$ Weights and Structure Coefficients
}

\section{Leily Ziglari}

Texas A \& M University

The importance of taking both $\beta$ weights and structure coefficients in interpreting regression studies, especially in applied linguistics papers, has often been ignored. The purpose of the present study was to explain both the regression coefficient and the structure coefficient to support the view that the inclusion of these two coefficients will augment the interpretation of results. Some examples of misinterpretations in regression studies were provided. Then the author applied both $\beta$ weights and structure coefficients in interpreting the results of an experimental study in applied linguistics. $\beta$ weights and structure coefficients were applied to investigate the predictive power of three predictors on the grammatical acquisition of a pair of twins. The results highlighted the importance of both coefficients in multiple regression studies.

$\mathrm{T}$ There are multiple reasons why graduate students need to use regression over traditional univariate analysis. Roughly 25 years ago, Thompson (1989) in one of his seminal papers addressed the superiority of regression analysis to ANOVA. Regression analysis as an important general linear model can be used both in experimental and non-experimental research study without mutilating independent variables into nominal scale - preventing the inflation of type II errors (Thompson \& Borrello, 1985; Thompson, in press; Zientek \& Thompson, 2009). Regression analysis can also be conducted for both continuous and categorical variables (Thompson, 1989), and it can do everything ANOVA can do, and more (Kerlinger \& Pedhazur, 1973). Cohen (1968) also argued using ANOVA to analyze experimental data reduces the reliability of most variables, and obliterates the most important information regarding the relationship among variables. Furthermore, Cohen noted that because all univariate parametric methods (i.e., ANOVA) are subsumed under the node of regression, they can be analyzed using regression. Moreover, Kieffer and Thompson (1999) criticized the significance of ANOVA by proposing a new method called "what if" analysis. What-if is a process through which the researcher can prospectively determine the sample size to obtain a statistically significant effect. Thus, the above-mentioned reasons are enough to motivate every graduate student to be curious about regression analysis, how it is implemented and interpreted.

The present study aimed to define and explain both $\beta$ weights and structure coefficients in interpreting multiple regression studies. As a graduate student, I found that researchers in applied linguistics (i.e., my field) infrequently reported $\beta$ weight and structure coefficients in their studies. A few others (e.g., Iwashita, 2003) only took $\beta$ weights and ignored structure coefficients. Two research questions were the focus of the current study:

1. What are $\beta$ weights and how are they interpreted?

2. What are structure coefficients and how are they interpreted?

\section{Multiple Regression and Collinearity}

\section{Literature Review}

Notwithstanding the above-mentioned reasons to advocate the multiple regression analysis in research, there is a condition of "collinearity" which makes interpreting regression results difficult (Kraha, Turner, Nimon, Zientek., \& Henson, 2012; Thompson, 2006a; Zientek \& Thompson, 2006). Collinearity is a condition in which "predictor variables have nonzero correlations with each other" (Thompson, 2006a, p. 236). Such a condition can "substantially affect the accuracy of interpretation of results" (Thompson \& Borrello, 1985, p. 204), and lead to "distortions in the estimations of regression coefficient or even to reversals in their signs" (Pedhazur, 1982, p.246). However, Thompson (2006a) does not consider collinearity a bad practice. Instead, he argues that such a complex model reflects the model of reality in that predictor variables coexist and affect outcome variables. Therefore, graduate students who are curious about linking their data analysis with the realistic models (Thompson, in press) should report statistic values for collinearity conditions in their research study.

Table 1 presents collinearity by invoking correlations among three predictors (X1, X2, X3). The result shows that the variables are collinear, and accordingly the amount of $\mathrm{R}^{2}$ obtained (i.e., shared 
variance) might be attributed to more than one predictor. However, Kraha, et al. (2012) noted that the correlation coefficient does not yield information about the shared explained variance.

As shown in Table 1, X1 has the strongest correlation with $\mathrm{Y}(\mathrm{r}=0.500)$ compared to $\mathrm{X} 2$ and $\mathrm{X} 3$. The squared correlation yields information that $25 \%\left(\mathrm{r}^{2}=0.25\right)$ of the outcome variance was explained by X1 (Kraha, et al., 2012). Also, 6.25\% $\left(\mathrm{r}^{2}=0.062\right)$ of the outcome variance was explained by $\mathrm{X} 3$ and no variance was explained by $\mathrm{X} 2$. In the following paragraph, two critical ways to interpret the strongest predictor compared to bivariate correlations have been provided.

\section{Critical Components in Multiple Regression Analysis}

It is critical to understand which coefficient (i.e., structure coefficient or regression coefficient) should be consulted in multiple regression analysis as an indicator "to determine the contribution of a variable to prediction" (Thompson \& Borrello, 1985, p. 207). Thompson and Borrello thoroughly explained the importance of structure coefficients compared to beta weight analysis $(\beta)$ after an effect size $\left(\mathrm{R}^{2}\right)$ is obtained.

$\mathrm{R}^{2}$ as a symbol of effect size in multiple regression studies addresses the question, "with knowledge of scores on the predictor variables, what proportion (or percentage) of the variability in individual differences on the outcome variable can we predict?" (Thompson, in press).

\section{Beta Weight in Regression Analysis}

There are two essential concepts about the importance of reporting beta weight in regression research analyses. First, regression is the univariate case of general linear model (GLM) (Cohen, 1968; Thompson \& Borrello, 1985; Zientek \& Thompson, 2009). Second, GLM means that all parametric analyses (a) are correlational, (b) apply weights to the measured variables to estimate score on synthetic variables, and (c) yield effect size analogous to $r^{2}$ values (e.g., Cohen; Thompson, 2006a). Keeping these two factors in mind, it is critical for the regression researchers not only to report the effect size value $\left(\mathrm{R}^{2}\right)$ and $\beta$ weights in their paper, but also thoroughly understand the meaning and conceptual logic behind them.

In this paragraph, the meaning and utility of the $\beta$ weight are explained. The beta weight $(\beta)$ means "given one unit of change in $\mathrm{Z}_{\mathrm{X} 1}$, how much Y-HAT will change" (Courville \& Thompson, 2001, p. 241). Explaining further, beta weights yield information about the extent to which a predictor is receiving credit for predicting the outcome variable in the regression equation, assuming other predictor variables held constant (e.g., Kraha, et al., 2012; Thompson, 2006a). Weights in regression are used to make Y and YHAT (predicted score) scores within a study match as closely as possible (Thompson, 2006a). They do this by making either means or standard deviations of two scores (Y, Y-HAT) match. Beta $(\beta)$ weights can be computed using the following formula (Thompson, 2006a, p. 235):

$$
\beta 1=\left[\mathrm{r}_{\mathrm{YxX1}}-\left\{\left(\mathrm{r}_{\mathrm{YxX} 2}\right)\left(\mathrm{r}_{\mathrm{X} 1 \times \mathrm{X} 2}\right)\right\}\right] /\left[1-\mathrm{r}_{\mathrm{Xx} 1 \mathrm{X} 2}{ }^{2}\right]
$$

For instance, $\beta 1$ can be computed as follows using the correlation matrix in Table 1 :

$$
\begin{aligned}
& \beta 1=\left[0.500-\{(0.000)(0.300\}] /\left[1-0.300^{2}\right]\right. \\
& \beta 1=0.517
\end{aligned}
$$

Kraha, et al. (2012) provided a summary table for multiple regression results using the correlation matrix in Table 1. The result was rendered in Table 2:

Table 1. Correlation matrix for classical suppression example

\begin{tabular}{cccc}
\hline & $\mathrm{Y}$ & $\mathrm{X} 1$ & $\mathrm{X} 2$ \\
\hline $\mathrm{Y}$ & 1.000 & & \\
$\mathrm{X} 1$ & 0.500 & 1.000 & \\
$\mathrm{X} 2$ & 0.000 & 0.300 & 1.000 \\
$\mathrm{X} 3$ & 0.250 & 0.250 & 0.250 \\
\hline
\end{tabular}

Table 2. Multiple Regression Results

\begin{tabular}{cccccccc}
\hline Predictor & $\beta$ & $\mathrm{r}_{\mathrm{s}}$ & $\mathrm{r}^{2} \mathrm{~s}$ & $\mathrm{r}$ & $\mathrm{R}^{2}$ & Unique & Common \\
\hline $\mathrm{X} 1$ & 0.517 & 0.911 & 0.830 & 0.500 & 0.250 & 0.234 & 0.016 \\
$\mathrm{X} 2$ & -0.198 & 0.000 & 0.000 & 0.000 & 0.000 & 0.034 & -0.034 \\
$\mathrm{X} 3$ & 0.170 & 0.455 & 0.207 & 0.250 & 0.063 & 0.026 & 0.037 \\
\hline
\end{tabular}

Note: $R^{2}=0.301 . r_{s}=$ structure coefficient $=r^{\prime} R ; r_{s=}^{2} r^{2} / R^{2}$; unique $=$ proportion of criterion variance explained uniquely by the predictor; common= proportion of criterion variance explained by the predictor that is also explained by one/more other predictors.

This table was adapted from Kraha et al. (2012). 
Given the formula of $\mathrm{R}^{2}$ (Thompson, 2006a), I calculated the squared multiple correlation coefficient using the $\beta$ weight and bivariate correlations in Tables 1 and 2 .

$$
\begin{aligned}
& \mathrm{R}^{2}=\beta_{1}\left(\mathrm{r}_{\mathrm{YX} 1}\right)+\beta_{2}\left(\mathrm{r}_{\mathrm{YX} 2}\right)+\ldots \ldots \beta_{\mathrm{p}}\left(\mathrm{r}_{\mathrm{YXP}}\right) . \\
& \mathrm{R}^{2}=\beta_{1}\left(\mathrm{r}_{\mathrm{YX} 1}\right)+\beta_{2}\left(\mathrm{r}_{\mathrm{YX} 2}\right)+\beta_{3}\left(\mathrm{r}_{\mathrm{YX} 3}\right) \\
& \mathrm{R}^{2}=0.517(0.500)+-0.196(0.000)+0.170(0.250) \\
& \mathrm{R}^{2}=0.2585+0.000+0.0420 \\
& \mathrm{R}^{2}=0.301
\end{aligned}
$$

$\mathrm{R}^{2}$ yields information about the explained variance of outcome variable $(\mathrm{Y})$. However, this amount might be attributed differently to different predictors. The $\beta$ weight yields information about the amount of credit each predictor received from $\mathrm{R}^{2}$. Predictor $\mathrm{X} 1$ received more credit $(\beta 1=0.517)$ compared to $\mathrm{X} 2$ $(\beta 2=-0.198)$ and $\mathrm{X} 3(\beta 3=0.170)$. Moreover, although $\mathrm{X} 2$ had a zero correlation with $\mathrm{Y}$, it received an amount of credit. It clearly shows that $\beta$ weights are not the same as correlation coefficient.

However, beta weight may be erroneously interpreted in regression studies. To interpret the result using $\beta$ weight, when predictors are perfectly uncorrelated, the $\beta$ weight yields the same information as the correlation coefficient ( $r$ ) does (Thompson, 1992b, 2006a). However, it might be mistakenly interpreted that near-zero beta weight does not have a predictive power because the pertinent product in $\mathrm{R}^{2}$ formula (i.e., formula 2) will be zero (Kraha et al., 2012) and therefore, it does not affect $\mathrm{R}^{2}$. Conversely, in collinearity conditions, the $\beta$ weight is not the same as correlation coefficient. Rather, it means how much credit each predictor is receiving in predicting outcome variable. It might be the case that the credit for shared or common explained variability in outcome variable was assigned to other predictors than to the predictor with near-zero $\beta$ weight.

In the light of foregoing, although $\beta$ weight is one of the tools that yields information about the predictive credit of each predictor, it has some features that affects the accuracy of interpretation. These features need to be considered in interpreting regression results: a) $\beta$ weight is not a sign of correlation coefficient, when predictors are correlated with each other (e.g., Thompson, 1992b, 2006a); b) beta weight equals each predictor's correlation with Y, if predictors are perfectly uncorrelated (Thompson, 2006a); c) a near-zero beta weight might have a high correlation with outcome variable, but the credit for the shared variance may have been assigned to other predictors (Zientek \& Thompson, 2006) (in this case, the variable with near-zero $\beta$ weight might be the single best predictor (e.g., Thompson, 1992b; Thompson \& Borrello, 1985)); and d) beta weight is context-dependent in that it is easily fluctuated as the correlation coefficient changes (Thompson, 1992b, 2006a), especially when sample size is small (Cooley $\&$ Lohnes, 1971). Furthermore, adding or deleting a predictor variable will change the beta weight's value and thus all the subsequent interpretations (Thompson, 1999b). Therefore, $\beta$ weights do not have generalizability power because they are context-dependent and; therefore, they are sample-specific weights (Cohen, 1968; Thompson, 2006a).

In sum, researchers need to conceptually understand both the meaning and interpretation of the $\beta$ weights. Such an understanding helps them know that even beta weights $(\beta)$ are not enough (Courville \& Thompson, 2001), and one needs to go further and reports structure coefficients $\left(\mathrm{r}_{\mathrm{s}}\right)$ as well (Thompson \& Borrello, 1985).

\section{Structure Coefficient $\left(\mathbf{r}_{\mathrm{s}}\right)$}

There are two essential reasons for the importance of structure coefficient in regression studies. A structure coefficient, as defined by Cooley and Lohnes (1971, p.55), "equals the bivariate correlation between the predictor and the criterion variable divided by the multiple correlation." Thompson (1999a) in one of his seminal papers stated that structure coefficients give much more insight regarding the nature of data dynamics. First, a structure coefficient is defined as the correlation between each predictor (i.e., X1) and Y-HAT (i.e., synthetic variable) or between each predictor and the whole eaten pie (Thompson, 1988, 2006a) - giving more insight into the synthetic variable. Second, structure coefficients give us better understanding about the predictive utility of the predictors (Courville \& Thompson, 2001; Thompson, 1988). Squared structure coefficient $\left(\mathrm{r}_{\mathrm{s}}{ }^{2}\right)$ gives us information about how much variance of $\mathrm{R}^{2}$ effect, the predictor can explain (Thompson, 1988, 1992b, 2006; Zientek \& Thompson, 2006). To put it another way, the squared structure coefficient yields the proportion of explained variance that each 
predictor alone explains (Kraha, et al., 2012). Therefore, the sum of squares of Y-HAT scores equals the regression or explained sum of squares (Thompson, in press).

Structure coefficients can be computed either using formula $r_{s}=r_{Y X 1} / R$ or $r_{s}=r_{X 1 Y-H A T}$ (Thompson, 2006a). Structure coefficients cannot be obtained through SPSS and can be calculated using Excel. In Table 3, I have provided the computation of

\begin{tabular}{|lll}
\hline \multicolumn{3}{|c}{$\begin{array}{l}\text { Table 3. Structure coefficient computation } \\
\text { for X1, X2, X3 }\end{array}$} \\
\hline \multicolumn{1}{c}{$\mathrm{X} 1$} & \multicolumn{1}{c}{$\mathrm{X} 2$} & \multicolumn{1}{c}{$\mathrm{X} 3$} \\
\hline $\mathrm{r}_{\mathrm{s}}=\mathrm{r}_{\mathrm{YX} 1} / \mathrm{R}$ & $\mathrm{r}_{\mathrm{s}}=\mathrm{r}_{\mathrm{YX} 2} / \mathrm{R}$ & $\mathrm{r}_{\mathrm{s}}=\mathrm{r}_{\mathrm{YX} 3} / \mathrm{R}$ \\
$\mathrm{r}_{\mathrm{s}}=0.500 / 0.548$ & $\mathrm{r}_{\mathrm{s}}=0 / 0.548$ & $\mathrm{r}_{\mathrm{s}}=0.250 / 0.548$ \\
$\mathrm{r}_{\mathrm{s}}=0.912$ & $\mathrm{r}_{\mathrm{s}}=0.000$ & $\mathrm{r}_{\mathrm{s}}=0.456$ \\
$\mathrm{r}_{\mathrm{s}=0.831 \%}^{2}$ & $\mathrm{r}_{\mathrm{s}=0.000}^{2}$ & $\mathrm{r}_{\mathrm{s}=0.207}^{2}$ \\
\hline
\end{tabular}

Note: $R=0.548 ; R^{2}=0.301$ structure coefficients and squared structure coefficients for three predictors $\mathrm{X} 1, \mathrm{X} 2$, and X3 using correlation matrix in Table 1.

As you see in Table 3, structure coefficient of X1 is represented as 0.912. Squared structure coefficient for X1 explains the amount of explained variance accounted for by X1 (i.e., $0.83 \%$ of $0.301=$ 0.250). This example clearly shows that the structure coefficient relates to the explained variance, not to the outcome variable as a whole.

In order to interpret which predictor has the strongest predictive power, Table 2 was consulted. The results suggested that predictor X1 has the strongest predictive power with reference to both $\beta$ weight and structure coefficient. X3 has the second strongest predictive power with structure coefficients of 0.455 . It explains $20.7 \%$ (i.e., $0.455^{2}$ ) of the explained variance (i.e., 0.301 ). On the other hand, although X2 has zero bivariate correlation ( $r$ ) with $\mathrm{Y}$ and zero structure coefficient $\left(\mathrm{r}_{\mathrm{s}}\right.$ ), it has, nevertheless, received credit in $\beta$ weight. This means that the given predictor has indirectly influenced other predictors in predicting the outcome variable. This is called a suppressor variable (Thompson, 2006a; Zientek \& Thompson, 2006). The present paper was not intended to explain suppresser variables, suffice it to say, that looking at both $\beta$ weights and structure coefficients helps us understand which predictor has strong predictive power.

However, interpreting the structure coefficient is determined conditionally: a) if $\beta$ weight and $r_{s}$ are both zero, the predictor variable is definitely useless; b) if $\beta$ weight is near-zero, we need to consult with $\mathrm{r}_{\mathrm{s}}$ to determine the importance of predictor variables; $\mathrm{c}$ ) if $\beta$ weight and bivariate correlation are the same, the predictors are uncorrelated; therefore, $\beta, \mathrm{r}_{\mathrm{s}}$ and $\mathrm{rs}$ will lead us to the same interpretation regarding the importance of the predictor; $d$ ) if $\beta$ weight is not the same as $r_{s}$, predictors are correlated, and both $\beta$ and $r_{s}$, need to be interpreted; e) if $r_{s}$ and $r s$ are both zero, one of the predictor variables is a suppressor variable (Zientek \& Thompson, 2006). A Suppressor variable is a predictor that has a large $\beta$ weight, but a zero correlation with the outcome variable. This affects the size of $R^{2}$ by making $\beta$ weights of other predictors deviate from the mathematical limit of +1 . In this case, $\beta$ does not represent the correlation coefficient because predictors are correlated with each other (i.e., collinearity). This condition is called the "suppressor effect" (e.g., Courville \& Thompson, 2001; Thompson, in press)

Notwithstanding the importance of structure coefficient, some researchers (e.g., Pedhazur, 1997) objected to using structure coefficients as the second tool in interpreting regression results. Pedhazur (1997) argued that large structure coefficients can also be obtained even when variables have near-zero $\mathrm{R}^{2}$ (i.e., results are meaningless). Thompson (1997a) explained:

When interpreting results in the context of this model, researchers should generally approach the analysis hierarchically, by asking two questions:

Do I have anything? (Researchers decide this question by looking at some combination of statistical significance tests, effect sizes, ... and replicability evidence.

If I have something, where do my effects originate? (researchers often consult both the standardized weights implicit in all analyses and structure coefficient to decide this question). (p.31)

However, it might be argued that structure coefficient, unlike $\beta$ weight, is not context-dependent and is not affected by collinearity. Thompson $(1988,2006)$ argued that context-independency of structure coefficients make them have generalizability power compared to $\beta$ weights. Therefore, in regression studies, both $\beta$ weights and structure coefficients need to be consulted for interpretation. In interpreting the results, $\beta$ weights are the credits given to each predictor for creating the predicted score, whereas structure coefficients are the bivariate correlation between each predictor and the effect observed (Burdenski, 2000; Kraha, et al., 2012). 


\section{Sample}

The current research investigation was a case study in that a sample of spontaneous language from twins' first language (L1) was video-taped. Data were elicited through interaction with a pair of 3-4 year old twin for a period of one year. The present study was aimed to examine the predictive power of positive and negative evidence of adult's talk on the grammatical correctness of subject-verb agreement in children's language acquisition. I used multiple regression analysis and interpreted the results by invoking both $\beta$ weights and structure coefficients.

\section{Method}

Adult-child interactions were videotaped and then, they were coded and transcribed by chat program available in CHILDES. Predictor variables were positive evidence and negative evidence. Positive evidence is when an adult (here, researcher) models the language on child's language production (e.g., simple model), whereas negative evidence is when adult corrects the child's grammatical error (Saxton, 2000). I categorized negative evidence into two types of clarification request and expansion, and I considered simple model as a type of positive evidence.

\section{Procedure}

The first step in the study of child language acquisition is collecting data and transcribing them. This brought about the problem that each researcher used an idiosyncratic coding system to transcribe data and the results could not be generalizable. The most powerful computational tool in this regard is CHILDES. CHILDES is a system through which language researchers can use or create the digitized data to study syntactic or morpho-syntactic development. MacWhinney and Snow (1985) created the CHILDES. I learned how to computerize my corpus data (i.e., transcribe, digitize, and analyze) under the supervision of Dr. Brian MacWhinney at Carnegie Melon University.

\section{CHILDES}

CHILDES is a system of computational tools for transcribing and analyzing data in language studies (MacWhinney, 2012). It was designed to automate the process of data analysis and increase the consistency of the transcription system. Although language acquisition research is grounded on the data collected from spontaneous interactions in naturally occurring situations, these descriptive data do not give us much information unless they are put into the process of transcribing and analyzing scientifically (MacWhinney). CHILDES as a computational process consists of CHAT, CLAN, and database. CHAT includes transcription and coding format, and describes the principles and conventions of transcription; CLAN includes analytic command to transcribe and analyze the interactions, and explains how to link digital audio or video files to transcripts (i.e. text); Database represents the accumulation of dozens of data files. It is worth mentioning to say that CHILDES system has wide applicability and is not necessarily limited to the first language acquisition field. A sample of the CHAT file is represented in the following (MacWhinney, p. 21).

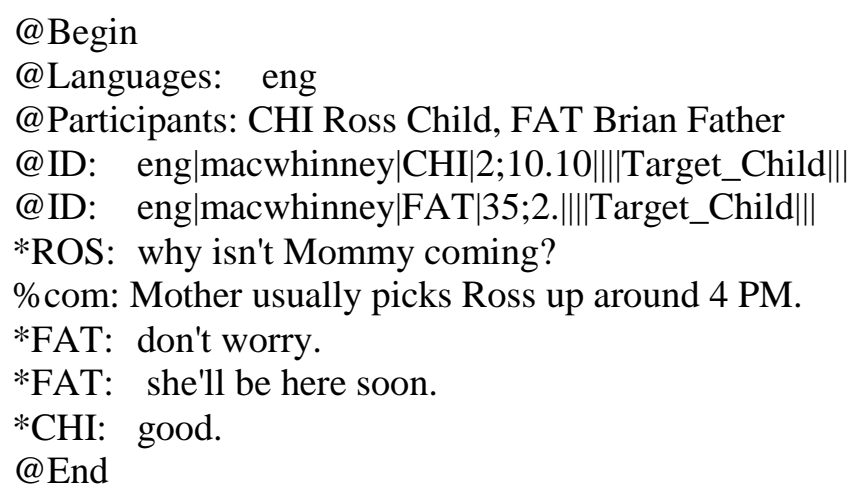

As shown in the above figure, this short dialogue was transcribed based on the CHAT principles in CHILDES system. Then, the researcher can obtain a command from CLAN program and run an analysis on this short dialogue. I transcribed all my data collection through CHAT program and obtained the frequency regarding the type of feedback the children received (i.e., positive evidence, negative evidence) in their language. I did this by invoking CLAN program. My corpus is available in CHILDES system for language researchers who need a corpus from adult-child interaction in Persian language. This corpus has 
made the job of future researchers easy. The researchers can use a few commands of CLAN program and analyze their research questions from a database that are already systematically transcribed and computerized into the CHILDES system.

The following clan commands were used in the present study of which their symbols are written as follows (MacWhinney, 2012):
a. CHECK Esc-L
b. CHIP Run chip +bMOT + +CCHI chip.cha
c. FREQ Run freq $+\mathrm{t} * \mathbf{C H I} 0042$.cha
d. MLU Run MLU +t*FAT 0042.cha

For instance, by typing item d, the researcher gives a command to the CLAN program to find Mean Length of utterance (i.e., MLU) of Fatima's speech in session 42 (i.e., 0042) among all utterances that Fatima spoke (i.e., +t*FAT), excluding all other participants. Accordingly, the bold words in each command can vary based on the target that the researcher wanted to investigate (i.e., linguistic feature, session, and participant).

\section{Results}

Multiple regression analysis is part of the GLM (Thompson, 2006a; Zientek \& Thompson, 2009), in that "all analytic methods are correlational... and yield variance-accounted-for effect sizes analogous to $r^{2}$ (e.g. $\mathrm{R}^{2}, \mathrm{y}^{2}, \omega^{2}$ )" (Thompson, 2000, p. 263). Therefore, researchers are required to include correlation matrices, standard deviations, group means, and sample size in their report so that the interested readers can conduct secondary analysis using available information (Zientek \& Thompson, 2009). Keeping this in mind, and with respect to the first research question, descriptive statistics rendered in Tables 4 and 5. Research question 1 asked what are beta weights and how are they interpreted?

A multiple regression analysis was used to obtain the amount of $\beta$ weight and $R^{2}$ in the present study. I already showed how $\beta$ weight is computed using correlation matrix in Table 1 (i.e., Formula 1). In this paragraph, I explained about the context-dependency of $\beta$ weights that makes the interpretation of results complex. Table 6 represents the summary of multiple regression analysis to interpret the results.

In order to interpret the $\beta$ weight, the researcher has to take the effect size into consideration. As shown in Table 6 , the effect size value was $21.2 \%$. In order to find the origin of the effect for each predictor variable, $\beta$ weight was consulted. The results of $\beta$ weight were obtained by using a multiple regression analysis. According to Table 6, simple model that was a type of positive evidence had the highest $\beta$ weight compared to other predictors, meaning it received more credit for predicting grammatical correctness.

As it was already noted, the $\beta$ weight is context-dependent and is easily affected by adding or deleting predictors. In order to clearly show this feature, I deleted one predictor from the set of predictors three times and each time, the amount of $\beta$ altered. Table 7 clearly represents different amounts of $\beta$ weight with reference to adding or deleting the predictors.

Although the amount of $\beta$ weight did not dramatically change, it might be due to the small number of data used in the present study. However, the sign of $\beta$ weight changed when expansion predictor was deleted from the set of predictors. According to Dr. Bruce Thompson (personal communication, April 27, 2015), it might be due to a suppresser effect. However, because the amount of $\mathrm{R}^{2}$ for the 3rd set of variables was only $14.6 \%$, the negative sign of $\beta$ weight is not a big challenge. Based on the abovementioned evidence, $\beta$ weight is not enough in interpreting multiple regression studies, and we need to consult structure coefficient as well.

The computation of structure coefficients helps us understand the meaning and interpretation behind them. Structure coefficients and squared structure coefficients are not obtained by using SPSS. In Table 3, I showed the computation of $r_{s}$ and $r_{s}^{2}$ using correlation matrix. The amount of the structure coefficient and squared structure coefficient for each predictor was presented in Table 6. The result of summary Table 6, supports the view that structure coefficient yields information about the data dynamics.

As shown in Table 6 , the amount of $\beta$ weight for predictor "simple model" was 0.364 . It means that $36.4 \%$ credit this predictor received from $21.2 \%$ of $\mathrm{R}^{2}$. The amount of structure coefficient for the same predictor was 0.826 , and the pertinent squared structure coefficient was 0.682 . The amount of 0.682 informs us that $68.2 \%$ of $21.2 \%$ predictor "simple model" alone explains. With regard to the predictor 
Table 4. Descriptive Statistics

\begin{tabular}{lccc}
\hline Variables & Mean & SD & $\begin{array}{c}\text { Number of } \\
\text { Sessions }\end{array}$ \\
\hline Grammatical & 47.60 & 6.998 & 100 \\
Correctness & & & \\
Simple Models & 34.63 & 7.884 & 100 \\
Confirmation & 16.24 & 3.082 & 100 \\
Expansion & 25.56 & 6.793 & 100 \\
\hline
\end{tabular}

Note. Number of children $=2$

"confirmation", the $\mathrm{r}_{\mathrm{s}}^{2}$ was 0.013. This predictor had both near-zero B weight and near-zero structure coefficient, it might be considered a "useless predictor". However, it cannot be strongly judged as a useless predictor, because the amount of $\mathrm{R}^{2}$ effect was only $21.2 \%$. With regard to the predictor "expansion" the $\beta$ weight received 0.271 credit in predicting the grammatical correctness. By looking at the structure coefficient, expansion predictor explained $37.3 \%$ of the $21.2 \%$. Therefore, taking both $\beta$ weight and $r_{\mathrm{s}}$ into account, the simple models predictor (e.g., positive evidence) was the strongest predictor compared to the expansion predictor (e.g., negative evidence), and confirmation was a useless predictor. However, because the size of $\mathrm{R}^{2}$ in the present study was only $21.2 \%$, it cannot be strongly interpreted that negative evidence was a useless predictor. I used this analysis to inform researchers in applied linguistics about the importance of both $\beta$ weights and structure coefficients in interpreting the multiple regression results.

\section{Discussion and Conclusion}

The present study was about the importance of both B weights and structure coefficients in interpreting multiple regression results. Analyzing and interpreting quantitative studies is "a critical acquired knowledge" for doctoral students. According to Thompson (1999b), doctoral curricula "seemingly have less and less room for quantitative statistics and measurement content, even while our knowledge base in these areas is burgeoning', (p. 24). Definitely, no thoughtful researcher wants to spend a considerable amount of time bearing all the tensions and challenges of carrying out a research project either financially or academically, and then come up with reporting the results using a statistic that does not contain a substantially significant value (i.e., $p$-value) (Thompson, 1992a). Many researchers in applied linguistics infrequently report the amount of both $\beta$ weights and structure coefficients in their regression studies, or they do not interpret the results correctly (e.g., Iwashita, 2003). The purpose of the current study was to answer two critical questions:

a. What are $\beta$ weights and how are they interpreted?

b. What are structure coefficients and how are they interpreted?

I explained that due to the "collinearity" condition among variables, correlation coefficient does not yield information about the predictive power of the predictor variables in multiple regression studies. On the other hand, $\mathrm{R}^{2}$ gives information about the explained variance of outcome variable. However, this amount might be attributed differently to different independent variables. Two tools help researchers obtain information about the origin of the effect. These are called $\beta$ weights and structure coefficients. However, $\beta$ weights are context-dependent and are easily affected by adding or deleting predictors. Structure coefficient, on the other hand, are not sensitive to the collinearity. Taken together, $\beta$ weights 
and structure coefficients give information about the data dynamics. I provided some examples to clearly show the importance of both $\beta$ weights and structure coefficients in interpreting regression results. Then I analyzed my own research data from a paper that I presented at SERA conference in 2015, taking into account both $\beta$ weights and structure coefficients.

I did this analysis focusing on the importance of both regression coefficients and structure coefficients with the hope that language researchers will come to understand that reporting these two statistic values would give them more information about the data dynamics. In the next paragraph, I provided some suggestions in using multiple regression studies.

\section{Suggestions}

In this paragraph, I have listed some suggestions in running regression studies.

1. Regression analysis has nothing to do with causal inferences; rather, it is the research design that makes causal inferences (Thompson, 1981). Therefore, multiple regression analysis can be used both in experimental and non-experimental research designs.

2. Regression analysis gives us better insight into the data dynamics by reporting both beta weights and structure coefficients. However, if researchers intend to only report multiple correlation coefficients in their studies, collinearity would not become a big challenge and they do not have to report the structure coefficient (Thompson \& Borrello, 1985).

3. Researchers are advised to gain more insight into standards of analyzing research data before publishing their papers by referring to seminal research resources and guidance (Skidmore \& Thompson, 2010; Thompson, 1995, 1999b, 2006b, 2008; Wilson, 1980).

4. Moreover, the way I analyzed my experimental research study (positive evidence vs. negative evidence) using $\beta$ weights and structure coefficients in this paper, can encourage language acquisition researchers to apply meta-analysis thinking. They can use multiple regression analysis on various similar studies and compare the results with the findings of the present paper.

5. Reporting correlation matrices, group means, standard deviations, and effect sizes in research studies would serve as building blocks for doing subsequent or secondary analysis, such as structural equation modeling or commonality analysis (Thompson, 1997b, 2006b; Zientek \& Thompson, 2006, 2009).

6 . Results of secondary study would shed the light on replicability of the study, whereas statistical significance of ANOVA does not give us information about replicability of the results (Carver, 1978; Thompson, 2001, 2006a; Thompson \& Kieffer, 2000).

7. Whenever we run a meta-analysis or a secondary analysis to examine previous research studies, using innovative research analysis, we need to observe respectful criticism throughout our scholarly dialogue (Thompson, 2008).

\section{References}

Azen, R., \& Budescu, D. V. (2003). The dominance analysis approach to comparing predictors in multiple regression. Psychological Methods, 8, 129-148.

Burdenski, T. K. (2000, January,). The importance of structure coefficients in multiple regression: A review with examples from published literature. Paper presented at the annual meeting of the Southwest Educational Research Association, Dallas.

Carver, R. C. (1978). The case against statistical significance testing. Harvard Educational Review, 48(3), 378-399.

Cohen, J. (1968). Multiple regression as a general data-analytic system. Psychological Bulletin, 70(6), 426-443.

Cooley, W. W., \& Lohnes, P. R. (1971). Multivariate data analysis. New York: John Wiley \& Sons.

Courville, T., \& Thompson, B. (2001). Use of structure coefficients in published multiple regression articles: beta is not enough. Educational and Psychological Measurement, 61(2), 229-248.

Iwashita, N. (2003). Negative feedback and positive evidence in task-based interaction: Differential effects on L2 development. SSLA, 25(1), 1-36.

Kerlinger, F. N., \& Pedhazur, E. J. (1973). Multiple regression in behavioral research. New York: Holt, Rinehart and Winston. 
Kieffer, K., \& Thompson, B. (1999, November,). Interpreting statistical significance test results: A proposed new "what if" method. Paper presented at the annual meeting of the Mid-South Educational Research Association, Pt. Clear, AL.

Kraha, A., Turner, H., Nimon, K., Zientek, L. R., \& Henson, R. K. (2012). Tools to support interpreting multiple regression in the face of multicollinearity. Frontiers in Psychology: Quantitative Psychology and Measurement, 3(44), 1-16.

MacWhinney, B. (2012). The CHILDS system. Retrieved from childs.psy.cmu.edu/intro/childs.pdf

MacWhinney, B., \& Snow, C. (1985). The child language data exchange system. Journal of Child Language, 12, 271-296.

Pedhazur, E. J. (1982). Multiple regression in behavioral research: Explanation and prediction (2nd ed.). New York: Holt. Rinehart and Winston.

Pedhazur, E. J. (1997). Multiple regression in behavioral research (3rd ed.). Ft. Worth, TX: Harcourt Brace.

Saxton, M. (2000). Negative evidence and negative feedback: Immediate effects on the grammaticality of child speech. First Language, 20, 221-252.

Skidmore, S. T., \& Thompson, B. (2010). Statistical techniques used in published articles: A historical review of reviews. Educational and Psychological Measurement, 70(5) 777-795.

Thompson, B. (1981, November). The problem of OVAism. Paper presented at the annual meeting of the Mid-South Educational Research Association, Lexington, KY.

Thompson, B. (1988, April). Canonical correlation analysis: An explanation with comments on correct practice. Paper presented at the annual meeting of the American Educational Research Association, New Orleans.

Thompson, B. (1989, January). Heuristics for understanding the concepts of interaction, polynomial trend, and the general linear model. Paper presented at the annual meeting of the Southwest Educational Research Association, Houston.

Thompson, B. (1992a). Misuse of ANCOVA and related "statistical control" procedures. Reading Psychology, 13(1), iii-xviii.

Thompson, B. (1992b, April). Interpreting regression results: Beta weights and structure coefficients are both important. Paper presented at the annual meeting of the American Educational Research Association, San Francisco.

Thompson, B. (1995). Publishing your research results: Some suggestions and counsel. Journal of Counseling and Development, 73, 342-345.

Thompson, B. (1997a). Editorial policies regarding statistical significance tests: Further comments. Educational Researcher, 26(5), 29-32.

Thompson, B. (1997b). The importance of structural coefficients in structural equation modeling confirmatory factor analysis. Educational and Psychological Measurement, 57, 5-19.

Thompson, B. (1999a, April). Common methodology mistakes in educational research, revisited, along with a primer on both effect sizes and the bootstrap. Invited address presented at the annual meeting of the American Educational Research Association, Montreal.

Thompson, B. (1999b). Five methodology errors in educational research: A pantheon of statistical significance and other faux pas. In B. Thompson (Ed.), Advances in social science methodology (vol. 5, pp. 23-86). Stanford, CT: JAI Press.

Thompson, B. (2000). Ten commandments of structural equation modeling. In L. Grimm \& P. Yarnold (Eds.), Reading and understanding more multivariate statistics (pp. 261-284). Washington, DC: American Psychological Association.

Thompson, B. (2001). Significance, effect sizes, stepwise methods, and other issues: Strong arguments move the field. Journal of Experimental Education, 70, 80-93.

Thompson, B. (2006a). Foundations of behavioral statistics: An insight-based approach. New York: Guilford.

Thompson, B. (2006b). Research synthesis: Effect sizes. In J. Green, G. Camilli, \& P.B. Elmore (Eds.), Handbook of complementary methods in education research (pp. 583-603). Washington, DC: American Educational Research Association.

Thompson, B. (2008). Standards in conducting and publishing research in education. Mid-Western Educational Researcher, 21(1), 10-16. 
Thompson, B. (in press). Multivariate statistics are vital. In B. Thompson (Ed.), Foundations of multivariate behavioral statistics. New York: Guilford.

Thompson, B., \& Borrello, G. M. (1985). The importance of structure coefficients in regression research. Educational and Psychological Measurement, 45, 203-209.

Thompson, B., \& Kieffer, K. M. (2000). Interpreting statistical significance test results: A proposed new "what if" method. Research in the Schools, 7(2), 3-10.

Willson, V. L. (1980). Research techniques in AERJ articles: 1969 to 1978. Educational Researcher, 9(6), 5-10.

Zientek, L. R., \& Thompson, B. (2006). Commonality analysis: Partitioning variance to facilitate better understanding of data. Journal of Early Intervention, 28(4), 299-307.

Zientek, L. R., \& Thompson, B. (2009). Matrix summaries improve research reports: Secondary analyses using published literature. Educational Researcher, 38(5), 343-352. 